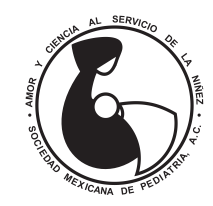

\title{
Reporte de caso de una niña con recurrencia de síndrome de Guillain-Barré
}

\author{
Ángel Solana-Rojas, ${ }^{1, *}$ Luis Miguel García-Melo, ${ }^{1}$ María Daniela Reyes-Varela, ${ }^{2}$ \\ Juan Francisco Díaz-Sotelo, ${ }^{1}$ Alfredo Cruz-Sánchez, ${ }^{1}$ Francisco Eduardo Basulto-López ${ }^{1}$ \\ ${ }^{1}$ Hospital Pediátrico Legaria; ${ }^{2}$ Hospital Pediátrico Coyoacán, Secretaría de Salud, Ciudad de México.
}

\begin{abstract}
RESUMEN
Introducción: El síndrome de Guillain-Barré (SGB) es una polirradiculoneuropatía desmielinizante. El síndrome de Guillain-Barré recurrente (RGBS) se presenta entre 1 y $6 \%$ de los pacientes, tras meses o años del primer episodio. Los casos en niños son infrecuentes. Objetivo: En este trabajo se presenta una escolar de siete años con RGBS. Descripción del caso: El primer episodio del SGB fue a los dos años; durante su estancia hospitalaria recibió inmunoglobulina intravenosa (IgIV). No hubo compromiso respiratorio y egresó a los pocos días por mejoría. El segundo episodio de SGB fue a los siete años, pero tuvo insuficiencia respiratoria, afección bulbar, arreflexia y disautonomías, requiriendo ventilación mecánica durante 37 días a pesar de tratamiento con IgIV. Por electromiografía se determinó SGB variante motor axonal aguda. Conclusiones: EI RGBS en niños es una variante rara. En la mayoría de los reportes el curso de los episodios subsecuentes es diferente al inicial.
\end{abstract}

Palabras clave: Síndrome de Guillain-Barré, recurrencia, electromiografía, niños.

\section{INTRODUCCIÓN}

El síndrome de Guillain-Barré (SGB) es una polirradiculoneuropatía desmielinizante aguda, de origen autoinmune que presenta diversas variantes clínicas. ${ }^{1}$ En la mayoría de los casos del SGB existe un cuadro infeccioso previo a las manifestaciones clínicas, las

\begin{abstract}
Introduction: Guillain-Barré syndrome (GBS) is a demyelinating polyradiculoneuropathy. Recurrent GuillainBarré syndrome (RGBS) occurs between 1 and $6 \%$, which appears after months or years of the first episode. Cases in children are uncommon. Objective: This paper presents a seven-year-old schoolgirl with RGBS. Case report: The first episode of the GBS was at two years of age; during her hospital stay, she received intravenous immunoglobulin (IVIG). There was no respiratory compromise; after improvement few days later, she left the hospital. The second episode of GBS was at seven years of age, but this time she had respiratory failure, bulbar disease, dysflexia and dysautonomies, requiring mechanical ventilation for 37 days, despite treatment with IVIG. By electromyography a SGB acute axonal motor variant was determined. Conclusions: RGBS in children is a rare variant. In most reports, the course of subsequent episodes is different from the initial one.
\end{abstract}

Keywords: Guillain-Barré syndrome, recurrence, electromyography, children.

cuales se caracterizan por parálisis aguda, debilidad progresiva de extremidades e incapacidad para la deambulación. . $^{1,2}$

En 1857, Landry describió los primeros casos del SGB. En 1916, Guillain-Barré y Strol demostraron que estos pacientes presentaban déficit motor y arreflexia, pero sin afección sensitiva y con disociación albúmino-

* Correspondencia: ASR, solanarojas.angel@gmail.com

Conflicto de intereses: Los autores declaran que no tienen.

Citar como: Solana-Rojas A, García-Melo LM, Reyes-Varela MD, Díaz-Sotelo JF, Cruz-Sánchez A, Basulto-López FE. Reporte de caso de una niña con recurrencia de síndrome de Guillain-Barré. Rev Mex Pediatr 2019; 86(6):238-242. doi: 10.35366/91876

[Case report of a girl with recurrence of Guillain-Barré syndrome] 
citológica. ${ }^{3,4}$ Mientras que, en 1990, Asbury y Comblath establecieron los criterios electrodiagnósticos del SGB, que se caracterizan por retraso en la velocidad de conducción de dos o más nervios motores. ${ }^{5}$

En la actualidad, el SGB es la causa más frecuente de parálisis flácida en niños previamente sanos. ${ }^{6} \mathrm{~A}$ nivel mundial la incidencia anual es de 0.6 a 2.4 casos por 100,000 habitantes. En cualquier grupo etario afecta a ambos géneros con una relación H/M 1.5:1. ${ }^{6}$ Se ha asociado con infección por C. jejuni, citomegalovirus, virus Epstein-Barr, virus influenza A, Mycoplasma pneumoniae y Haemophilus influenzae. ${ }^{7}$

Existen distintas formas de presentación del SGB: el SGB clásico, la debilidad faringo-cérvico-braquial, SGB paraparético y la debilidad facial bilateral con parestesias. Mientras que, del síndrome de Miller Fisher (SMF), también puede distinguirse el SMF incompleto y la encefalitis de Bickerstaff. En el año 2014, The GBS Classification Group describió los criterios para su identificación. 8,9

En cuanto al tratamiento, desde hace años se conoce la efectividad de la inmunoglobulina intravenosa (IgIV) y la plasmaféresis. En pacientes pediátricos, la IgIV se puede aplicar de dos maneras: en la primera (más efectiva), la dosis es $1 \mathrm{~g} / \mathrm{Kg}$ de peso por día, en dos días. La segunda, la dosis es de $0.4 \mathrm{~g} / \mathrm{Kg}$ de peso, administrada en cinco días. ${ }^{2,10}$

El síndrome de Guillain-Barré recurrente (RGBS, por sus siglas en inglés), ocurre entre 1 y $6 \%$ de los pacientes; se ha informado que se presenta de varios meses a años a partir del primer episodio de SGB hasta en $10 \%$ de los pacientes tras un periodo de estar asintomático. ${ }^{11,12}$ Entre los factores de riesgo de RGBS se encuentran: edad menor de 30 años y presentación previa de síndrome de Miller Fisher. ${ }^{12}$ En general, hay pocos casos reportados de RGBS en niños. ${ }^{11-13}$

Se presenta el caso de una niña de siete años con RGBS después de cinco años de haber presentado el primer episodio de SGB, con énfasis en la evolución clínica progresiva, afección neurológica y en las complicaciones.

\section{PRESENTACIÓN DEL CASO}

\section{Primer evento de SGB}

Paciente de dos años de edad que acudió a urgencias debido a que, después de un proceso infeccioso de vías respiratorias superiores, y una semana previa a su ingreso se agregaron síntomas clínicos del SGB, caracterizados por debilidad en ambas extremidades inferiores.
A su ingreso se observaron signos vitales normales: FC 116/minuto, FR 30/minuto, Temp $36^{\circ}$ C, saturación de oxígeno 93\%. Exploración física: femenino de edad aparente similar a la cronológica, adecuada hidratación, cráneo normocéfalo, cavidad oral con hipertrofia amigdalina grado II y faringe hiperémica, cuello sin megalias, cardiopulmonar sin compromiso, abdomen blando sin megalias ni irritación peritoneal, extremidades superiores con fuerza conservada $5 / 5$ en escala de Daniels, reflejos osteotendinosos conservados, retiro al dolor y sensibilidad conservada. En las extremidades inferiores se identificó disminución de la fuerza $3 / 5$ en escala de Daniels, arreflexia bilateral; pero había retiro de las mismas al dolor y la sensibilidad estaba conservada. Neurológico: despierta y reactiva a estímulos externos, marcha no valorable, pares craneales conservados, signos meníngeos ausentes y sin disautonomías.

\section{Evolución clínica hospitalaria}

Día 1. Exámenes de laboratorio: biometría hemática: leucocitos $6,800 \mathrm{leu} / \mu \mathrm{L}$, neutrófilos $27 \%$, linfocitos $62 \%$, monocitos $8 \%$, hemoglobina $13.6 \%$, hematocrito $40.3 \%$, plaquetas $482 \mathrm{mil}$, PCR $0 \mathrm{mg} / \mathrm{dL}$. Electrolitos séricos: sodio $138 \mathrm{meq} / \mathrm{L}$, potasio $4.9 \mathrm{meq} / \mathrm{L}$, cloro $105 \mathrm{meq} / \mathrm{L}$, calcio $11.1 \mathrm{mg} / \mathrm{dL}$, fósforo $6.2 \mathrm{mg} / \mathrm{dL}$, magnesio $2.3 \mathrm{mg} /$ dL. Glucosa $70 \mathrm{mg} / \mathrm{dL}$, BUN $22 \mathrm{mg} / \mathrm{dL}$, urea $47 \mathrm{mg} / \mathrm{dL}$, creatinina $0.3 \mathrm{mg} / \mathrm{dL}$. CPK $737 \mathrm{U} / \mathrm{L}$ (valores normales en niñas entre dos y tres años: 10-120 U/L)

Características del líquido cefalorraquídeo (LCR): claro, incoloro, transparente, células 1: monocitos $100 \%$, eritrocitos negativos, glucosa $43 \mathrm{mg} / \mathrm{dL}$, cloruro $118.3 \mathrm{meq} / \mathrm{L}$, proteínas $57 \mathrm{mg} / \mathrm{dL}$, Pandy positivo (+), $\mathrm{pH}$ 7.6, lactato 1.6. No se observan bacterias; coaglutinación negativa.

Se inició tratamiento médico con inmunoglobulina intravenosa (IgIV) a dosis de $1 \mathrm{~g} / \mathrm{Kg}$, por tres días.

Día 2. Estudio de neuroconducción: SGB variante desmielinizante inflamatoria aguda (Figura 1).

Día 3. Cultivo de líquido cefalorraquídeo negativo. Hemocultivo periférico negativo.

Día 6. Durante su estancia hospitalaria presentó adecuada evolución, sin complicaciones secundarias, adecuada tolerancia a la vía oral, sin dificultad respiratoria y sin ameritar apoyo ventilatorio. Además, hubo mejoría paulatina en la movilidad de extremidades inferiores: fuerza 4/5, arreflexia bilateral, sensibilidad conservada. Se decidió su egreso con apoyo de silla de ruedas y envío a rehabilitación pediátrica por seis meses. 
Median MNC

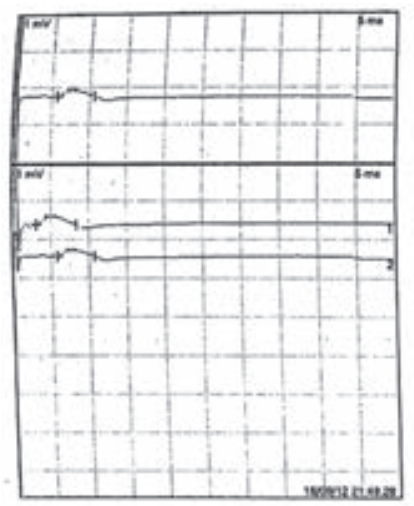

Sural SNC

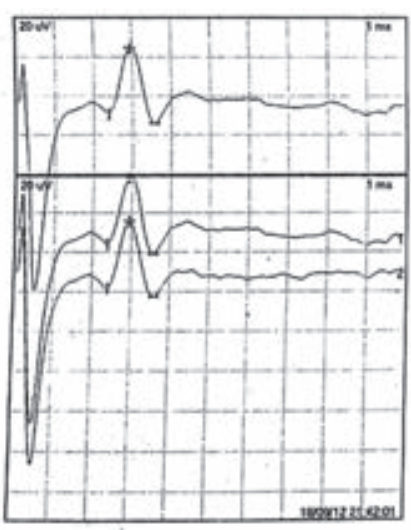

Tibial MNC

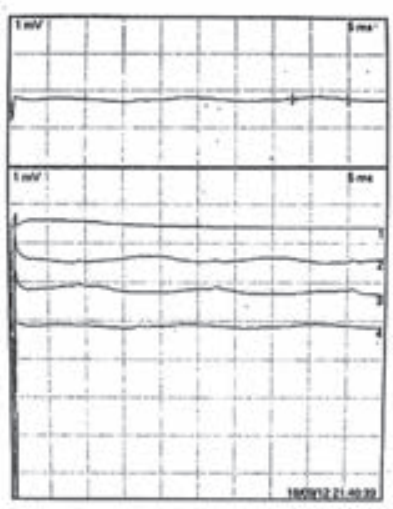

L. Median F-wave

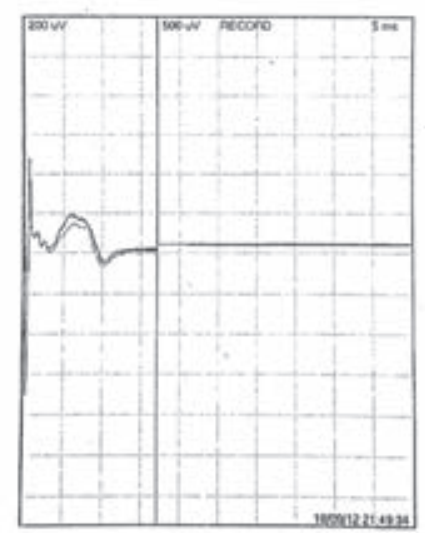

L. Median SNC

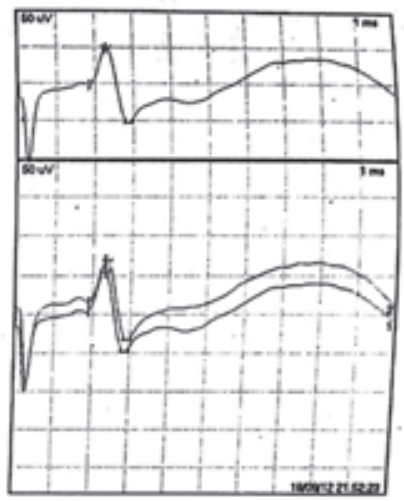

Figura 1:

Estudio de neuroconducción. (Femenino de dos años).

Interpretación. Estudio de neuroconducción con respuesta anormal, nervio periférico distal y proximal con retraso en la conducción compatible con SGB variante desmielinizante inflamatoria aguda.

\section{Segundo evento de SGB}

Femenino de siete años de edad. El padecimiento actual consistió en un cuadro de infección de vías aéreas superiores de tres días de evolución. Se agregaron parestesias de miembros inferiores, las cuales fueron ascendentes, progresivas y simétricas, llegando hasta extremidades superiores. Además, se observó mal manejo de secreciones bronquiales, por lo cual fue llevada al servicio de urgencias para su valoración.

A su ingreso los signos vitales fueron: FC 95/minuto, FR 14/minuto, saturación de oxígeno 93\%, temperatura $36.5^{\circ}$ C. Exploración física: adecuado estado hídrico, ruidos cardiacos normales al igual que la auscultación pulmonar; el abdomen sin megalias ni datos de irritación peritoneal. Las extremidades superiores con fuerza disminuida 2/5 en escala de Daniels, reflejos osteotendinosos abolidos y sensibilidad conservada. En las extremidades inferiores también hubo disminución de la fuerza 0/5 en escala de Daniels, arreflexia bilateral, no se retiraban al dolor, pero la sensibilidad se conservó. La paciente se observaba despierta y reactiva a estímulos externos, sin ptosis palpebral, pupilas isocóricas y reflejos a la luz normales; fondo de ojo normal, pares craneales sin alteraciones, funciones mentales superiores conservadas, marcha no valorable, signos meníngeos ausentes y sin disautonomías.

Se diagnosticó SGB, por lo cual se decidió hospitalizar.

\section{Evolución clínica hospitalaria}

Semana 1 (días 1-7). Durante sus primeras horas en el servicio de urgencias presentó dificultad respiratoria, por lo cual se decidió manejo avanzado de la vía aérea e ingreso al servicio de terapia intensiva. Por la rápida progresión del cuadro neurológico se decidió inicio de IgIV a dosis de $1 \mathrm{~g} / \mathrm{kg}$ por dos días. Se mantuvo con apoyo mecánico ventilatorio con intubación orotraqueal, manteniendo sedación con midazolam; ante la falta de mejoría clínica y ausencia de movimientos respiratorios espontáneos se realizó traqueostomía. 
Exámenes de laboratorio. Biometría hemática: leucocitos 14,140 leu/ $\mu \mathrm{L}$, neutrófilos 59.6\%, linfocitos $29.7 \%$, hemoglobina $14.8 \%$, hematocrito $44.2 \%$, plaquetas 434,000 , VSG $20 \mathrm{~mm} / \mathrm{h}$, procalcitonina $0 \mathrm{ng} /$ $\mathrm{mL}$. CPK 1236 U/L (valores normales: 50-145 U/L).

Se llevó a cabo evaluación por el servicio de neurología pediátrica; en su nota señalan que la paciente se encuentra bajo efecto de sedación, las pupilas isocóricas hiporrefléxicas, con tendencia a la miosis. Simetría facial. La fuerza en extremidades superiores proximal $2 / 5$ y distal $1 / 5$; extremidades inferiores, fuerza proximal y distal $0 / 5$. Reflejos tricipital y bicipital disminuidos; mientras que los reflejos patelar y aquíleo están ausentes de manera bilateral. Respuesta plantar flexora, no clonus, sensibilidad conservada, retiro al dolor.

Se decidió realización de punción lumbar; el resultado de LCR fue: líquido claro, incoloro, transparente, dos células (monocitos), eritrocitos negativos, glucosa $54 \mathrm{mg} / \mathrm{dL}$, cloruro $110 \mathrm{meq} / \mathrm{L}$, proteínas $103 \mathrm{mg} / \mathrm{dL}$,

\begin{tabular}{lcccc} 
& \multicolumn{4}{c}{$\begin{array}{c}\text { Conducción motora } \\
\text { Nervio peroneo bilateral }\end{array}$} \\
& Tibial derecho & Tibial izquierdo & Mediano derecho & Mediano izquierdo \\
\hline Latencias & $7.1 \mathrm{~ms}$ & $7.7 \mathrm{~ms}$ & No valorado \\
Amplitud & $0.1 \mathrm{mV}$ & $0.5 \mathrm{mV}$ & $3.8-8.7 \mathrm{~ms}$ & $0.1 \mathrm{mV}$ \\
Velocidad & & & $37 \mathrm{~m} / \mathrm{s}$ &
\end{tabular}

\begin{tabular}{lcc} 
& Conducción sensitiva \\
& Nervio sural bilateral & \\
& Sural derecho & Sural izquierdo \\
\hline Latencias & $2.2 \mathrm{~ms}$ & $2.4 \mathrm{~ms}$ \\
Amplitud & $51 \mathrm{Uv}$ & $46 \mathrm{uV}$ \\
Velocidad & $45 \mathrm{~m} / \mathrm{s}$ & $42 \mathrm{~m} / \mathrm{s}$
\end{tabular}

\begin{tabular}{lll} 
& \multicolumn{2}{c}{$\begin{array}{c}\text { Onda F } \\
\text { Nervio tibial posterior bilateral }\end{array}$} \\
& Tibial derecho & Tibial izquierdo \\
\hline Latencias & No obtenido & No obtenido \\
Reclutamiento & &
\end{tabular}

Sural SNC

Tibial MNC
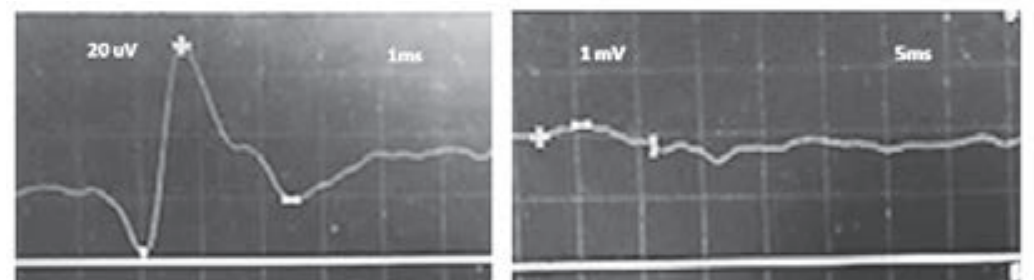

Figura 2:
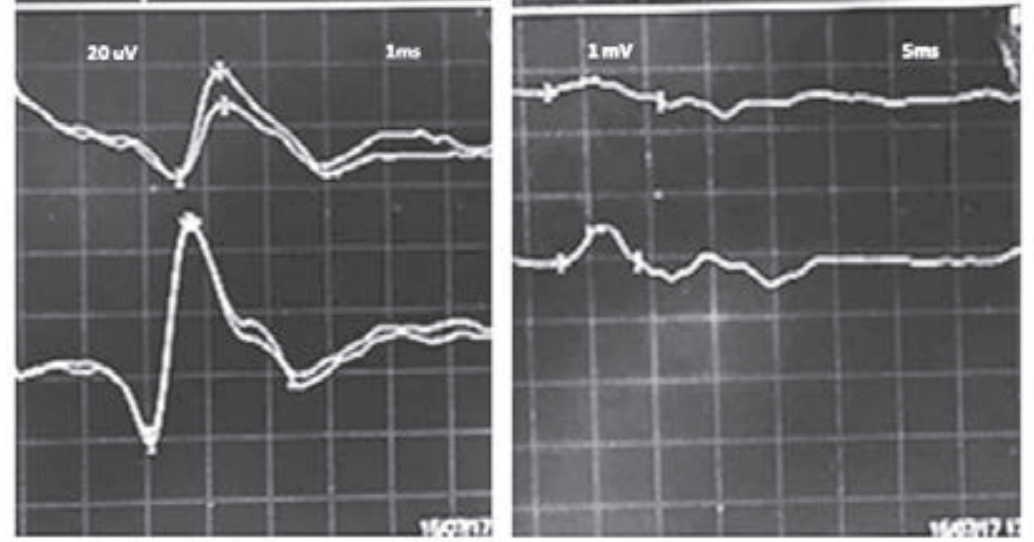

Estudio de neuroconducción. (Femenino de siete años).

Interpretación. Estudio de neuroconducción motora de nervio peroneo, sin obtención de respuesta. Latencia distal de nervio tibial prolongada, baja amplitud y dispersión temporal. Respuesta motora de nervio mediano disminuida en amplitud y retraso en conducción con similar dispersión de la respuesta. Onda F no obtenida. Sólo se preserva la respuesta sensitiva con presencia de retraso en su conducción. SGB con componente axonal. 
Pandy positivo (+), pH 7.6, lactato 1.4, no se observan bacterias, y la coaglutinación es negativa.

TAC de cráneo simple y contrastada: sin alteraciones estructurales y/o reforzamientos anormales.

Se solicitó realización de estudio de neuroconducción. Reporte de estudio: anormal sugestivo de SGB, con componente axonal (Figura 2).

Semana 2 (días 8-14). Se retiró sedación. La paciente tuvo comunicación verbal escasa y se inició aporte vía oral basado en líquidos claros con adecuada tolerancia. Reflejo tusígeno ausente.

Semana 3 (días 15-21). Progresión de alimentación con dieta polimérica y posteriormente dieta blanda con adecuada tolerancia.

Semana 4 (días 22-28). Inicio de dieta normal. Evolución de forma paulatina con mejoría clínica en la movilidad de la extremidad superior derecha y cintura escapular.

Semana 5 (días 29-35). Incremento de movilidad de ambas manos, y pies en dorsiflexión realiza pronosupinación. Incremento en la movilidad de la cintura escapular bilateral y cadera.

Semana 6 (días 36-42). Se retiró ventilación asistida y hubo apoyo de oxígeno suplementario; sin datos de dificultad respiratoria.

Semana 7 (día 43). Exploración neurológica: evolución favorable, fuerza 3/5 en escala de Daniels en extremidades superiores; en extremidades inferiores la fuerza 1/5 en escala de Daniels (se clasifica en escala de Hughes IV, paciente confinado en cama o silla sin capacidad de realizar marcha). No se detectó compromiso respiratorio y los reflejos de tallo cerebral están conservados.

La paciente es egresada con manejo externo en rehabilitación.

Cuatro meses. En la consulta de seguimiento de neurología pediátrica se observó recuperación gradual del SGB, obteniendo una calificación de escala de Hughes III, dado a que posee capacidad de caminar al menos 5 metros con ayuda de caminador y asistida por sus padres. Se decide continuar con tratamiento médico y sesiones de rehabilitación.

\section{DISCUSIÓN}

La RGBS es una entidad rara que se presenta hasta en $6 \%$ de todos los pacientes con SBG. ${ }^{14}$ Sólo hay algunas publicaciones que incluyen niños con RGBS. ${ }^{11,12}$ En estas series los pacientes mostraron presentaciones diversas y, en un buen número, se ha observado recuperación rápida en comparación al caso que describimos.
En nuestro caso de RGBS se observaron características diferentes a las descritas en otros estudios: (1) el patrón inusual de debilidad en la nueva presentación, (2) fenotipo neuropático motor axonal en el estudio de neuroconducción, (3) respuesta lenta y progresiva posterior a la administración de inmunoglobulina intravenosa requiriendo asistencia a la ventilación por semanas. Esta forma de presentación se parece a un caso reportado en el año 2013 en Estados Unidos de Norteamérica. ${ }^{11}$ En otro caso en Honduras se describe el comportamiento de un adolescente en tres episodios de SGB. ${ }^{15}$

\section{REFERENCIAS}

1. Arnason GB, Soliven V. Acute inflammatory demyelinating poliradiculoneuropathy. In: Dick PJ, Thomas PK, Griffin JW, Low PA, Podulso JF (eds). Peripheral neuropathy. 3th ed. Philadelphia: WB Saunders; 1993. pp. 1437-1497.

2. Agrawal S, Peake d, Whitehouse WP. Management of children with Guillain-Barré syndrome. Arch Dis Child Educ Pract Ed. 2007; 92(6): 161-168.

3. Landry O. Note sur la paralyse ascendante aigué. Gas Hebd Med Chir. 1859; 2: 472-474.

4. Guillain G, Barré JA, Strohl A. Sur un syndrome de radiculoneurite avec hyperalbunimose du liquide céphaloraquidien sans reactivon cellulaire Remarques sur les caractères cliniques et graphiques des reflexes tendineux. Bull Soc Med Hôp Paris. 1916; 40: 146-170.

5. Asbury AK, Cornblath DR. Assessment of current diagnostic criteria for Guillain-Barré syndrome. Ann Neurol. 1990; 27(suppl): S21, S24.

6. Jones $\mathrm{Hr}$ Jr. Guillain-Barré syndrome: perspectives with infants and children. Semin Pediatr Neurol. 2000; 7(2): 91-102.

7. Islam Z, Jacobs BC, van Belkum A, Mohammad $Q D$, Islam MB, Herbrink $P$ et al. Axonal variant of Guillain-Barré syndrome associated with campylobacter infection in Bangladesh. Neurology. 2010; 74 (7): 581-587.

8. Wakerley BR, Uncini A, Yuki N, GBS Classification Group. GuillainBarré and Miller Fisher syndromes-new diagnostic classification. Nat Rev Neurol. 2014;10:537-544.

9. Rebolledo-García D, González-Vargas PO, Salgado-Calderón I. Síndrome de Guillain-Barré: viejos y nuevos conceptos. Med Int Méx. 2018;34 (1): 72-81.

10. Hughes RA, Cornblath DR. Guillain-Barré syndrome. Lancet. 2005; 366: 1653-1666.

11. Dy M, Leshner RL, Crawford JR. An unusual case of recurrent Guillain-Barre syndrome of a different subtype five years after initial diagnosis. Case Rep Neurol Med. 2013; 2013: 356157. doi: 10.1155/2013/356157.

12. Grand'Maison F, Feasby TE, Hahn AF, Koopman WJ. Recurrent Guillain-Barré syndrome. Clinical and laboratory features. Brain. 1992; 115(Pt 4): 1093-1106.

13. Mossberg N, Nordin M, Movitz C, Nilsson S, Hellstrand K, Bergström $T$ et al. The recurrent Guillain-Barré syndrome: a long-term population-based study. Acta Neurol Scand. 2012; 126(3): 154-161.

14. Das A, Kalita J, Misra UK. Recurrent Guillain Barré syndrome. Electromyogr Clin Neurophysiol. 2004; 44(2): 95-102.

15. Oliva MG, Ramos FC. Síndrome de Guillain Barré Recurrente: Reporte de caso. Acta Pediatr Hondureña. 2008; 8 (2): 791-795. 